\title{
New Generation of IP-Phone Enabled Mobile Devices
}

\author{
Michimune Kohno ${ }^{1}$ and Jun Rekimoto ${ }^{1}$ \\ Interaction Laboratory, Sony Computer Science Laboratories, Inc., \\ 3-14-13 Takanawa-Muse Bldg., Higashi-Gotanda, Shinagawa-ku, \\ Tokyo, 141-0022 Japan \\ $\{$ mkohno, rekimoto\}@csl.sony.co.jp
}

\begin{abstract}
This paper describes how IP-phone communication and realworld user interface can become a new standard for mobile terminals. Current wireless broadband networks such as the $802.11 \mathrm{a} / \mathrm{b}$ will eventually lead to IP mobile phones. As "smart" appliances emerge on the market, mobile terminals can play a new role in the ubiquitous computing environment. This paper integrates IP-phone communication capability and intuitive user interface into a mobile terminal. It explains the use of the "pick-and-drop" technique as a controlling interface for voice sessions and music streams, enabling both usability and security, important in a practical ubiquitous computing environment. Finally, a prototype implementation will be briefly described.
\end{abstract}

\section{Introduction}

The rapid development of computer networks provides us with the ability to communicate with one another, wired or wireless, all over the world through broadband network in the forms of web technology, VPN, and host-spot services.

Information appliances will soon emerge on the market by introducing protocols such as INS, UPnP, and Jini[1,2]. The main purpose of these protocols is to control appliances remotely by using PCs, PDAs, or mobile phones.

Mobile devices receive broadband network communication capability through IEEE $802.11 \mathrm{a} / \mathrm{b}$. As wireless infrastructure (such as hot-spot locations) increases, more users are expected to access private data from a remote site with mobile devices in the near future.

A mobile device with network communication can be an IP-phone terminal device using current VoIP technologies[3], which allows us to make phone calls without carrier lines. Despite obstacles with mobility support[4], fast handover, and power consumption, integrating voice communication into a mobile terminal will add to its value by combining the conveniences of a mobile phone and a computer to create a new generation of handheld devices.

This paper describes the effects of this terminal in a ubiquitous computing environment, presents some practical uses, and proposes a solution. 


\section{Mobile Device with Voice Communication Capability}

The fusion of a mobile phone and a computer goes beyond the idea that the two is mounted on a single component. For instance, provided with the current technology, when sending a file to the person on the receiving end of a phone call, you have to attach it to an e-mail with the correct e-mail address by using the mail service on your PC. However, if the PC has telephone capability, no e-mail address needs to be selected because the $P C$ is already connected to the receiver through the phone call. Ideally, IP voice communication does not limit the number of callers, allowing the capability to collectively send data to all callers easily.

Although this feature exists in many instant messaging applications, it has yet to be applied to mobile devices. Furthermore, these applications do not offer any functions to access private data stored at a remote site.

By meshing appliances and mobile terminals, new applications for current technologies such as projectors, tablet-type computers, audio devices, and car gadgets will emerge for the ubiquitous computing environment.

The following scenarios show concrete applications of this technology:

- You are talking with a mobile phone while walking. You can transfer the call to the microphone and speaker in the car or office.

- You have just received a file sent by a peer. You can display it on a nearby projector.

- You are listening to music in your room with a stereo. You can transfer the music session to your mobile phone and go out.

Although security problems and access control rights may arise in these example scenarios, which are important issues for practical use, this paper only focuses on user interface that provides usability for such applications. We will discuss security issues in another paper.

\section{User Interface Technique as Session Control}

Several approaches can be taken to utilize a mobile terminal as a universal remote control in a ubiquitous computing environment; however, most work in this area focus on how to display the controlling interface on a window $[5,6]$.

This paper proposes a different approach from the existing research by:

- integrating the IP-phone function, and

- using the pick-and-drop technique[7] as the user interface for the session control

The user interface technique that Rekimoto et al originally proposed uses direct manipulation for transferring files between computers. We adopted it to switch the receiving end in a voice communication or a music stream from one 


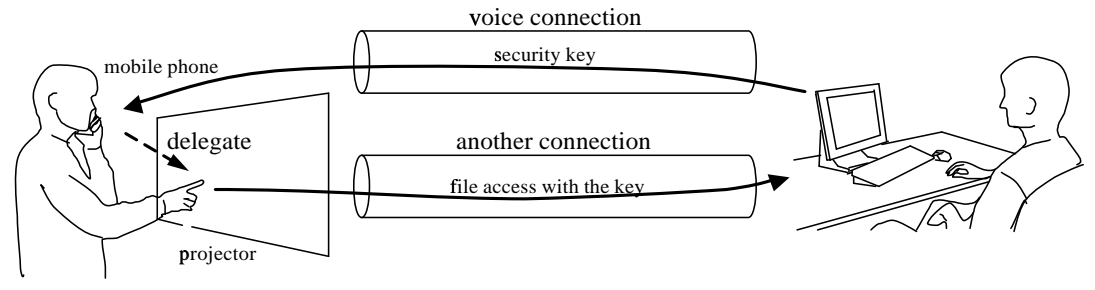

Fig. 1. An example scenario: using a mobile phone as a controlling interface for a wall projector. By bringing the mobile phone closer to the wall, the contents of the received file is projected on the wall. Note that security credentials are implicitly transferred to grant access rights to the file

device to another, namely, physically bringing the terminals closer together by mobilizing the endpoint of a session. In a similar way, if you want to see a received file on a certain display, you can bring the mobile terminal closer to the display. The display would then grant temporary access rights to the file allowing the viewer to open the file. The viewing device can either be controlled via the terminal or the opened voice session.

As mobile phones merge with computer networks in the future, the mobile terminal will become increasingly important as a controlling device in the ubiquitous computing environment. This characteristic is further enhanced by adding such intuitive user interfaces as having the capability to physically bring objects closer, pointing at a device (like a remote control), and so on. Furthermore, from a technical perspective, it is important that such intuitive user interfaces can implicitly resolve security issues that usually degrade usability.

\section{Prototype Implementation}

We have created a prototype incorporating the characteristics. Fig.2 illustrates the overview. We used a Linux PDA as the handheld device with an 802.11b network interface card. We then implemented voice communication capability using a simple protocol and H.323. This same system was also implemented running on Windows2000.

We used UPnP as the underlying protocol. Therefore if a terminal enters an area in which wireless communication is available, any appliance with this type of communication enabled can be detected by the terminal.

As the target appliance, we used a PC with speakers and a microphone. In order to detect the approach of an existing terminal to the speakers, an RFID tag and a reader/writer device were used. The reader/writer, which is connected to the PC via RS232C, was attached to the speaker, and an RFID 


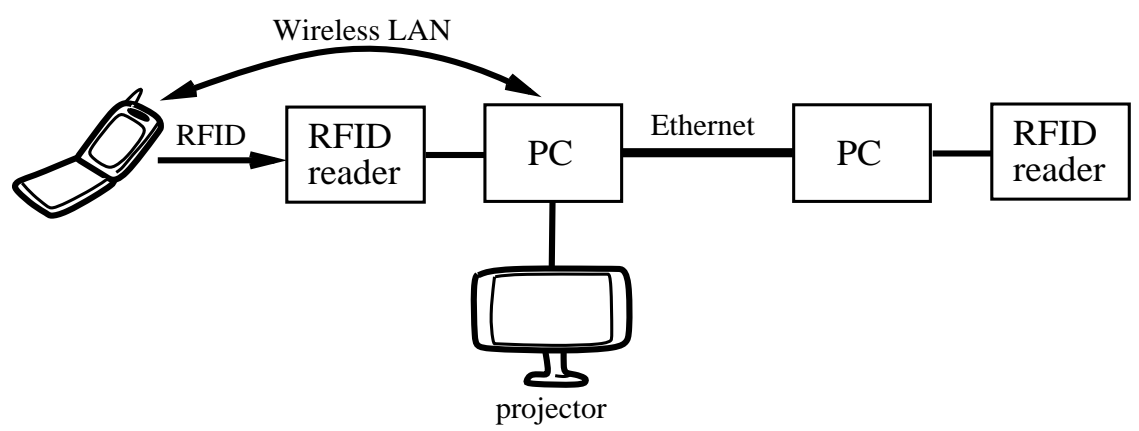

Fig. 2. The prototype system overview

tag was attached to the terminal. The detection of the RFID tag activates the session control procedure, which opens communication between the PC and the terminal.

RSA, a popular public key encryption mechanism, is used to verify security information. When communication is opened by detecting the RFID tag, digital certificates are exchanged with each other and verified. If authentication is correct, then a secured session channel is opened between the PC and the terminal to prevent other appliances from accessing. Next, the PC gives the terminal temporary access rights via the secured session.

Thus, this system allows us to open a connection without touching the LCD screen of the terminal.

\section{Conclusion}

This paper explained how voice communication and real-world user interface can create a new generation of mobile terminals, which can also implicitly resolve security issues.

Passing security information in a session with voice communication already established is still a fairly new concept, and critical for mobile devices to become actual controllers of smart appliances.

We believe that numerous applications that combine with the ubiquitous computing environment will emerge in the near future, thus we plan to develop more practical systems. The details of how such systems can become more secure will be discussed in another paper.

\section{References}

1. Adjie-Winoto, W., Schwartz, E., Balakrishnan, H. and Lilley, J.: The design and implementation of an intentional naming system, In Proc. of the 17th ACM SOSP, Dec. 1999. 
2. Microsoft Corportaion: Understanding Universal Plug and Play: A White Paper, http : //www . upnp.org/download/UPNP_UnderstandingUPNP.doc.

3. IETF: Session Initiation Protocol, http://www.cs.columbia.edu/sip/.

4. Perkins, C. and Johnson, B.: Mobility Support in IPv6, in Proc. of MOBICOM'96, ACM, 1996.

5. Buyukkokten, O., Garcia-Molina, H., Paepcke, A. and Winograd, T.: Power Browser: Efficient Web Browsing for PDAs, in Proc. CHI2000, Apr. 2000.

6. Zimmermann, G., Vanderheiden, G. and Gilman, A.: Prototype Implementations for a Universal Remote Console Specification, in Proc. CHI2002, Apr. 2002.

7. Rekimoto, J.: Pick-and-Drop: A Direct Manipulation Technique for Multiple Computer Environments, in Proc. of UIST'98, ACM Press, pp. 31-39. 